\title{
A boxy bulge in the Milky Way. Inversion of the stellar statistics equation with 2MASS data
}

\author{
M. López-Corredoira ${ }^{1,2}$, A. Cabrera-Lavers ${ }^{1}$, and O. E. Gerhard ${ }^{2}$ \\ ${ }^{1}$ Instituto de Astrofísica de Canarias, C/.vía Láctea, s/n, 38200 La Laguna (S/C de Tenerife), Spain \\ e-mail: [martinlc;acabrera]@iac.es \\ 2 Astronomisches Institut der Universität Basel, Venusstrasse 7, 4102 Binningen, Switzerland \\ Received 27 January 2004 / Accepted 31 March 2005
}

\begin{abstract}
Inverting the stellar statistics equation from 2MASS star counts, we obtain the 3D density distribution of the Galactic bulge as well as its luminosity function in the $K$-band. This results in a boxy bulge with axial ratios 1:0.5:0.4 and a major axis angle with respect to the Sun-galactic center of $20^{\circ}-35^{\circ}$.
\end{abstract}

Key words. Galaxy: structure - infrared: stars

\section{Introduction}

Due to extinction, near-infrared (NIR) surveys are the most appropriate observations to study the stellar population of the Galactic central components. However, we see them edge-on, so the task of constraining their parameters is not as easy as in other external galaxies, which can be observed face-on. In order to recover the 3D information, López-Corredoira et al. (2000; hereafter L00) developed a method of inversion of the star counts based on Bayesian statistics, which permits the obtaining of both the stellar density distribution and the luminosity function (LF) without making any a priori assumptions about them.

The L00 inversion technique has been applied to the $K$-band TMGS star counts up to $m_{K}=9.0$ in some strips that crossed the Galactic plane, mostly in positive longitudes (L00). The inversion was also applied to mid-infrared MSX Galactic plane maps (López-Corredoira et al. 2001a), although in this last case there were very few stars to derive the stellar density and only the LF could be derived with some accuracy. Now, we have a deeper and larger coverage (the whole sky) survey: 2MASS, with 3 NIR filters (which permits extinction correction) as well as some improvements on the disc models (necessary to be subtracted from the total star counts and isolate the bulge counts), so we have the chance to obtain more precise results from the bulge density distribution and its LF. This is the goal of the present paper: using the L00 inversion technique with the newest data and information.

\section{2MASS star counts and disc subtraction}

The 2MASS survey provides magnitudes of all stars in the NIR filters $H, K_{\mathrm{s}}$ in the range $7.5<m_{H}<15.1,7.0<$ $m_{K}<14.3$ and nearly one magnitude less in the galactic plane
(Skrutskie et al. 1997). It also provides J magnitudes, but we will not use these here. We can build maps of cumulative star counts within these ranges (Alard 2001). In order to make an approximate correction of the extinction (Alard 2001), we calculate

$m_{\mathrm{e}}=m_{K}-1.77(H-K)$,

and we produce maps of cumulative star counts with $m_{\mathrm{e}}<m_{\mathrm{e}, 0}$ and $m_{\mathrm{e}, 0}$ between 7.0 and 10.75 in steps of $0.25 \mathrm{mag}$. The angular space resolution both in longitude and latitude is $0.5^{\circ}$ in the range $-20^{\circ}<l<20^{\circ},-12^{\circ}<b<12^{\circ}$.

In order to isolate the bulge star counts, we must first subtract the disc star counts in the regions where both disc and bulge contribute along the line of sight. We cannot apply the inversion of L00 to disc+bulge counts since both components have different LFs and, moreover, the L00 method does not work for stars near the Sun as the relative error of the kernel in the inversion becomes very large.

The disc counts are given in López-Corredoira et al. (2004), interpreted as a truncated and/or flared disc:

Disc model 1: An exponential function of the galactocentric distance $(R)$ for $R>4 \mathrm{kpc}$ :

$$
\begin{aligned}
& \rho=\rho_{\odot} \mathrm{e}^{-\left(\frac{R-R_{\odot}}{1970 \mathrm{pc}}\right)} \mathrm{e}^{-\frac{k t}{h_{z}(R)}} \mathrm{star} \mathrm{pc}^{-3}, \\
& h_{z}(R)=285 \mathrm{e}^{\left(\frac{R-R_{\odot}}{(112-0.6 R(\mathrm{kp})] \mathrm{kp} c}\right)} \mathrm{pc},
\end{aligned}
$$

and a constant density for $R \leq 4 \mathrm{kpc}$ :

$$
\begin{aligned}
& \rho=5.1 \rho_{\odot} \mathrm{e}^{-\frac{|z|}{h_{z}(R)}}{\operatorname{star~} \mathrm{pc}^{-3}} \\
& h_{z}(R)=509-48 R(\mathrm{kpc}) \mathrm{pc} .
\end{aligned}
$$


Disc model 2: A hole in the inner disc which can be modeled $\forall R<R_{\odot}$ as:

$\rho=\left[\rho_{\odot} \mathrm{e}^{\left(\frac{R_{\odot}}{1970 \mathrm{pc}}+\frac{3740 \mathrm{pc}}{R_{\odot}}\right)}\right] \mathrm{e}^{-\left(\frac{R}{1970 \mathrm{pc}}+\frac{3740 \mathrm{pc}}{R}\right)} \mathrm{e}^{-\frac{|z|}{h_{z}(R)}} \operatorname{star}^{\mathrm{pc}^{-3}}$,

$$
\begin{aligned}
h_{z}(R)= & 285\left[1+0.21 \mathrm{kpc}^{-1}\left(R-R_{\odot}\right)\right. \\
& \left.+0.056 \mathrm{kpc}^{-2}\left(R-R_{\odot}\right)^{2}\right] \mathrm{pc} .
\end{aligned}
$$

In all cases we adopt $R_{\odot}=7.9 \mathrm{kpc}$. Indeed both models are almost equivalent for $2.5 \mathrm{kpc}<R<R_{\odot}$. They differ substantially only for $R<2.5 \mathrm{kpc}$. We do not know the exact disc profile in this region where it is mixed with the bulge, although we know that it lies between model 1 and 2 .

The disc LF used in the $K$-band is that given by Eaton et al. (1984). In order to obtain the correspondence between $m_{K}$ and $m_{\mathrm{e}}$, through Eq. (1), we calibrate $\left\langle(H-K)_{0}\right\rangle$ (color in regions without extinction) in a region towards the center where the disc is almost isolated and without extinction $\left(l=20^{\circ}, b=\right.$ $6^{\circ}$ ), as a function of $m_{\mathrm{e}, 0}$. In the regions $-20.25^{\circ}<l<-17.75^{\circ}$, $3.75^{\circ}<|b|<12.25^{\circ}$, we calibrate the amplitude of the density which gives the best fit: $\rho_{\odot}=0.050 \mathrm{star}_{\mathrm{pc}}{ }^{-3}$ for both models. This is close to the value used in López-Corredoira et al. (2004).

The results of the disc subtraction using model 1 are shown in Fig. 1 (Total - disc) for $m_{\mathrm{e}}<9.5$; for different $m_{\mathrm{e}, 0}$ the results are similar.

\section{Inversion of bulge star counts}

The fitting of parameters of the density and the LF in the bulge would be a way to carry out the analysis, but many free parameters would be needed, especially for the LF, and a knowledge of the shape of both functions is required. The L00 inversion technique is not limited in this way. However, some assumptions are needed: the disc models in Sect. 2 and the reddening law of Eq. (1); the assumption of constant LF throughout the bulge, and that the noise effects in the inversion do not significantly change the solution (as tested).

The procedure to invert the stellar statistics equation once the disc is subtracted is described in L00. It is an iteration of consecutive Lucy iterations. The LFs in $K$-band and the density for each line of sight are obtained as numerical functions; nevertheless, we fit "a posteriori" the parameters of an analytical 3-D function for the density of a triaxial bulge, and we use a weighted average of LFs in the different lines of sight. The result of each individual iteration does not depend significantly on the initial guess and, in the final global outcome, the average/fit of all the lines of sights, the dependence on the initial guesses is even smaller, much smaller than other sources of error and dispersion with respect to the average/fit.

There is a wrong statement in L00. $R_{\odot}$ cannot be determined by this method, because any factor in the distance scale can be compensated for by making the absolute magnitudes of all stars fainter or brighter; we recently realized that the value $R_{\odot}=7.9 \mathrm{kpc}$ given by L00 was indeed obtained because of the selected range of $M_{K}$ in the algorithm. This value was obtained by chance. However, the rest of the parameters are independent

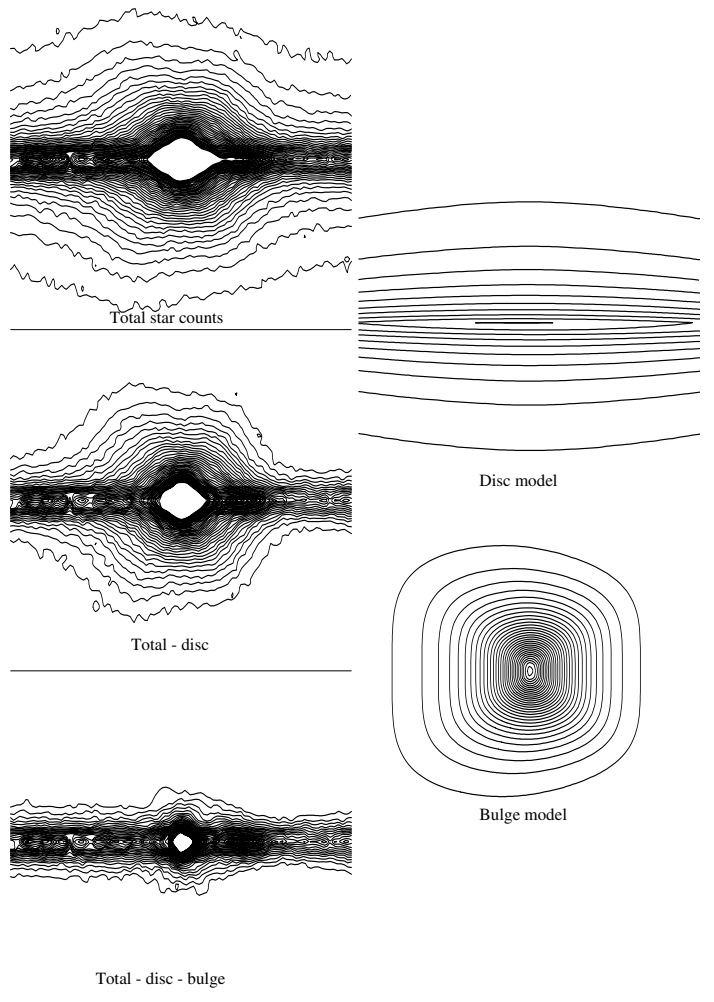

Fig. 1. Star counts maps with $m_{\mathrm{e}}<9.5$, for the region $-20^{\circ}<l<20^{\circ}$, $-12^{\circ}<b<12^{\circ}$. "Total" map stands for the counts derived from 2MASS. "Disc" map is the prediction of the disc "model 1" contribution (see Sect. 2). "Total-disc" is the subtraction of the disc in the total counts map. "Bulge" stands for the best triaxial boxy-isodensity fit of the result of the inversion (see Sect. 4) of "Total-disc". "Total-discbulge" are the residuals after subtracting the bulge from the "Totaldisc" map. The isocounts contours represent, from the outermost to the innermost: 600 to $30000 \mathrm{star} \mathrm{deg}^{-2}$ with steps of $600 \mathrm{star} \mathrm{deg}^{-2}$.

of the assumed value of $R_{\odot}$; it only affects the horizontal calibration of the LF, and, since $R_{\odot}=7.9 \mathrm{kpc}$ is in agreement with other measures, the presented results in $\mathrm{L} 00$ should be correct. Thus, we continue to adopt $R_{\odot}=7.9 \mathrm{kpc}$.

We repeat here the steps explained by L00 but with the present 2MASS data instead of TMGS data. The selected region is $-20^{\circ}<l<20^{\circ}, 2.25^{\circ}<|b|<12.25^{\circ}$; we avoid the Galactic plane, where other possible sources apart from the disc and bulge may be present. The subtracted disc model is different from that of L00 (see Sect. 2). A major improvement is that we do not need to specify the extinction, but we can correct the star counts by means of Eq. (1). By doing this, we avoid the possible problems with the patchiness of the extinction. The selected range of magnitudes to obtain the 3D-density is: $7.00<m_{\mathrm{e}, 0}<9.50$. For the LF, the inversion is less sensitive to the errors, so we have further extended the range up to $m_{\mathrm{e}, 0}<10.75$. One cannot extend the fainter limit too much because the ratio bulge/disc becomes poorer. The normalization of the LF is made assuming the value given by L00 for $\phi\left(M_{K}=-6.4\right)=2.34 \times 10^{-5}$. We get two outcomes: the LF in the range between $M_{K}=-6.6$ and $M_{K}=-2.8$, and the 3D-density distribution of the bulge. 


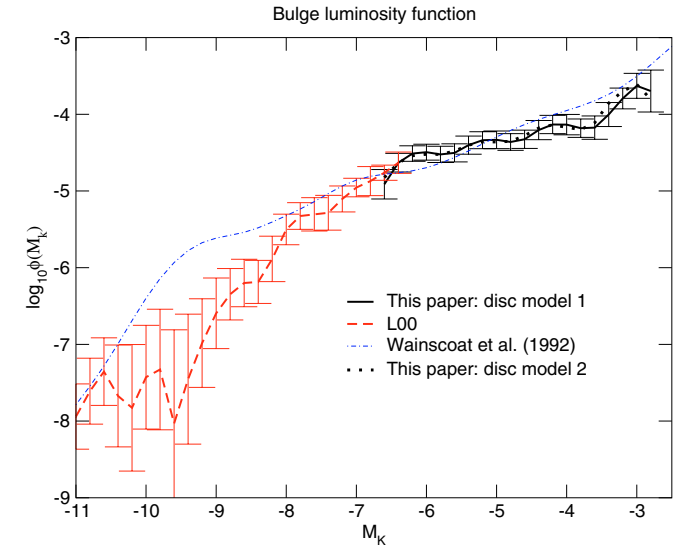

Fig. 2. Luminosity function derived in this paper (with disc models 1 and 2; error bars correspond to model 1 and are similar for model 2) and L00 in comparison with the synthetic LF of Wainscoat et al. (1992). Assumed $R_{\odot}=7.9 \mathrm{kpc}$.

Figure 2 shows the LF, with the extension to brighter stars derived from the TMGS by L00. In total, we have a LF over 8 magnitudes. A comparison with Wainscoat et al.'s (1992) synthetic LF (derived from a model of stellar populations) shows that there is a strong deficit of very bright stars $\left(M_{K}<\right.$ $-8)$ - supergiants and late $M$ giants - with respect to the synthetic $\mathrm{LF}(\mathrm{L00})$, and slight differences around $M_{K}=-6$ and $M_{K}=-3.5$.

Figure 3 shows different cuts of the 3D density distribution with $z=$ constant, obtained when using model 1 of the disc. Model 2 of the disc gives similar results (see Fig. 4). This figure gives the smoothed raw result from the L00 inversion technique, $\rho(\boldsymbol{r})$, and clearly shows the non-circularity of the contours in the bulge cuts. This confirms that the bulge is nonaxisymmetric, with its elongated structure closer to us in the first quadrant $(l>0)$.

If we assume that the bulge is constituted by triaxial ellipsoids, their parameters would be as follows: For the model 1 of the disc, the fitted mean axial ratios are 1:0.49:0.37; the angle between the major axis of the bulge (in the first quadrant) and the line Sun-Galactic center: $\alpha=29^{\circ}$; and the density profile $\rho(t) \approx 6.6 \mathrm{e}^{-\frac{t}{740 \mathrm{pc}}} \mathrm{star}_{\mathrm{pc}}^{-3}$, with $t=$ $\sqrt{x_{1}^{2}+\left(x_{2} / 0.49\right)^{2}+\left(x_{3} / 0.37\right)^{2}}$ where $x_{i}, i=1,2,3$ are along the major, middle and minor axis of the triaxial ellipsoid, respectively. Tests to recover a given 3D-density/LF with known parameters applying the whole L00-method to its projected star counts show that typical systematic deviations due only to the inversion method and noise are: $90 \mathrm{pc}$ for the scale length of the profile, $8^{\circ}$ for the angle, and 0.04 for both axial ratios. For the model 2 of the disc, the mean axial ratios are 1:0.49:0.40, angle $\alpha=27^{\circ}$.

The bulge is closer to axisymmetry (ratios 1:1:x) than 1:0.33:0.22 (Dwek et al. 1995), 1:0.38:0.26 (Freudenreich 1998) or 1:(0.3-0.4):0.3 (Bissantz \& Gerhard 2002). However, it is not very far from the values of 1:0.6:0.4 (Binney et al. 1997), 1:0.43:0.29 (Stanek et al. 1997) or 1:0.54:0.33 (L00). A lower ellipticity is more in accord with other galaxies, because bulges generally do not have large asymmetries. This also

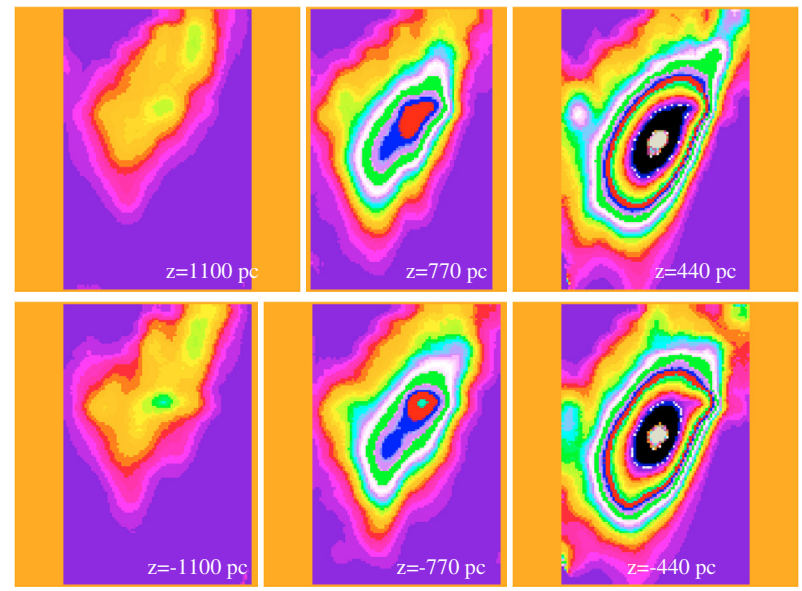

Fig. 3. Different cuts $(z= \pm 440, \pm 770, \pm 1100$ pc $)$ through the $3 \mathrm{D}$ bulge density distribution, obtained from the bulge counts with the L00 inversion technique after subtracting the model 1 for the disc. Vertical axis, parallel to the line Sun-Galactic center, between 4.4 and $11.0 \mathrm{kpc}$ from the Sun; increased distance from the Sun upwards. Horizontal axis, perpendicular to the line Sun-Galactic center: between -2.2 and $2.2 \mathrm{kpc}$; positive longitude to the left. $R_{\odot}=7.9 \mathrm{kpc}$. The contours represent isodensity regions; the density range between

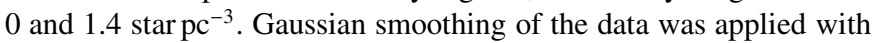
$\sigma_{x}=\sigma_{y}=220 \mathrm{pc}, \sigma_{z}( \pm 440 \mathrm{pc})=55 \mathrm{pc}, \sigma_{z}( \pm 770 \mathrm{pc})=110 \mathrm{pc}$, $\sigma_{z}( \pm 1100 \mathrm{pc})=110 \mathrm{pc}$.

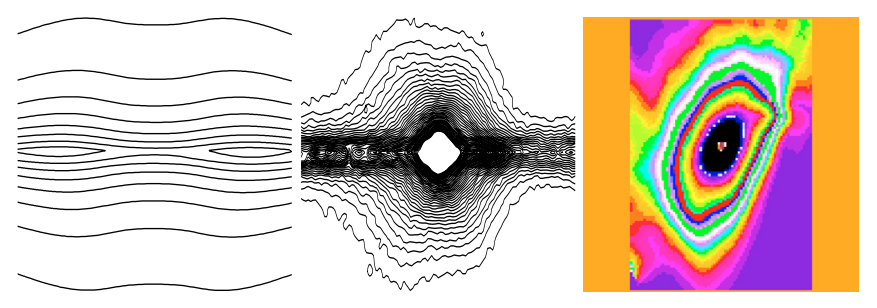

Fig. 4. Left: star counts of the disc "model 2" contribution (see Sect. 2); isocounts contours like in Fig. 1. Middle: "Total-disc" with "model 2". Right: cut at $z=-440$ pc as in Fig. 3 but with "model 2" for the disc.

contributes to clarify the difference between a bulge and a bar in our Galaxy. The present structure looks like a bulge rather than a stick-like bar. We suspect that the derived bulge axial ratios are different due to the dependence of the methods to correct for extinction and the disc subtraction. In our case we used NIR star counts corrected for extinction based on reddening, and an inner truncation and/or flare in the disc model increases the number of stars associated with the bulge, which reduces its global axisymmetry. The result might also depend on the method of inversion/fitting and the a priori assumptions.

A parameter which seems to be in agreement with most other studies is the orientation of the major axis of the bulge (when they refer to the bulge and not to the hypothetical inplane bar; see López-Corredoira et al. 2001b): $12^{\circ}-30^{\circ}$ (Dwek et al. 1995; Nikolaev \& Weinberg 1997; Stanek et al. 1997; Binney et al. 1997; Freudenreich 1998; Sevenster et al. 1999; L00; Bissantz \& Gerhard 2002). 


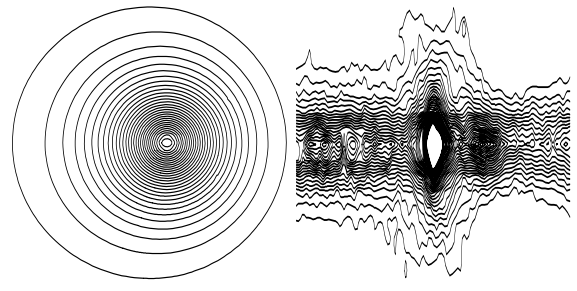

Fig. 5. Left: best triaxial ellipsoid fit of the result of the inversion (see Sect. 3) of "Total-disc" in Fig. 1. Right: "Total-disc-bulge" map. Isocount contours as in Fig. 1.

\section{Boxy bulge}

Although a triaxial ellipsoid represents the bulge in first order, the shape of the projection ("Total-disc" in Fig. 1) is boxier than the projection of ellipsoids (Fig. 5). The boxy shape of the bulge was noted previously (Kent et al. 1991, 1992; Dwek et al. 1995). Here, the boxiness is even more remarkable than in previous papers, due perhaps to a different subtraction of the disc and the availability of star counts instead of flux maps. The isodensity contours in Fig. 3, especially those at higher $t$ and smaller $z$, look boxier also than an ellipse. If we use $t=\left[x^{4}+\left(x_{2} / a\right)^{4}+\left(x_{3} / b\right)^{4}\right]^{1 / 4}-$ a similar boxy structure as was proposed by Kent et al. (1991) for the axisymmetric case - we get a better representation with somewhat fewer residuals (Fig. 1). For this boxy-model, we adopt the same angle $\alpha$ and axial ratios, and the same profile $\rho(t)$ as for the ellipsoids but with $t_{\text {boxy }}=0.866 t_{\text {ellip }}$. This is the approximation that gives the best correspondence between ellipsoids and boxy-isocontours with the same inclination and axial ratios. The integrated boxycontours do not perfectly represent the observed isocount contours in the outmost regions - it seems that the corners are not so abrupt - but they give a better model than the elliptical bulge. The asymmetry of the counts in the residuals in plane regions (Fig. 1, "total-disc-bulge") suggests that part of these remaining stars are due to another axisymmetric component, presumably a long bar. Other components like a ring, spiral arms, and errors in the disc subtraction and extinction correction in plane regions are also expected to contribute to the residuals.

\section{Discussion and conclusions}

It is well known that boxy bulges are related to bars in edge-on galaxies (Merrifield \& Kuijken 1999). Their bar-like nature is revealed by the spectroscopical data which show the typical dynamics of multiple-component line emission with one of the components being bar-like. Our Galaxy has one of these boxy-bulges, which is not itself long-bar-like but quite thick: axial ratios 1:0.5:0.4. Therefore, the scenario of an in-plane bar apart from the bulge (López-Corredoira et al. 2001b; Hammersley et al. 2001, and references therein) is supported. The asymmetry of the residuals "Total-disc-bulge" also agrees with this scenario. The triaxial bulge has an angle $\alpha \approx 20^{\circ}-35^{\circ}$ with respect to the line Galactic center-Sun, smaller than the long-bar angle.

Acknowledgements. Thanks to the anonymous referee and Peter Englmaier for helpful comments. This publication makes use of data products from 2MASS, which is a joint project of the Univ. of Massachusetts and the Infrared Processing and Analysis Center (IPAC), funded by the NASA and the NSF. We thank the Swiss National Science Foundation for support under grant 20-64856.01.

\section{References}

Alard, C. 2001, A\&A, 379, L44

Binney, J., Gerhard, O. E., \& Spergel, D. 1997, MNRAS, 288, 365

Bissantz, N., \& Gerhard, O. E. 2002, MNRAS, 330, 591

Dwek, E., Arendt, R. G., Hauser, M. G., et al. 1995 ApJ, 445, 716

Eaton, N., Adams, D. J., \& Gilels, A. B. 1984, MNRAS, 208, 241

Freudenreich, H. T. 1998, ApJ, 492, 495

Hammersley, P. L., López-Corredoira, M., \& Garzón, F. 2001, in Tetons 4: Galactic Structure, Stars and the Interstellar Medium, ed. C. E. Woodward, M. D. Bicay, J. M. Shull (S. Francisco: ASP), ASP Conf. Ser., 231, 81

Kent, S. M., Dame, T. M., \& Fazio, G. 1991, ApJ, 378, 131

Kent, S. M., Mink, D., Fazio, G., et al. 1992, ApJS, 78, 403

López-Corredoira, M., Hammersley, P. L., Garzón, F., Simonneau, E., \& Mahoney, T. J. 2000, MNRAS, 313, 392 (L00)

López-Corredoira, M., Cohen, M., \& Hammersley, P. L. 2001a, A\&A, 367, 106

López-Corredoira, M., Hammersley, P. L., Garzón, F., et al. 2001b, A\&A, 373, 139

López-Corredoira, M., Cabrera-Lavers, A., Gerhard, O. E., \& Garzón, F. 2004, A\&A, 421, 953

Merrifield, M. R., \& Kuijken, K. 1999, A\&A, 345, L47

Nikolaev, S., \& Weinberg, M. D. 1997, ApJ, 487, 885

Sevenster, M., Prasenjit, S., Valls-Gabaud, D., \& Fux, R. 1999, MNRAS, 307, 584

Skrutskie, M. F., Schneider, S. E., Stiening, R., et al. 1997, in The Impact of Large Scale Near-IR Sky Surveys, ed. F. Garzón, N. Epchtein, A. Omont, B. Burton, \& P. Persi (Dordrecht: Kluwer), 25

Stanek, K. Z., Udalski, A., Szymański, M., et al. 1997, ApJ, 477, 163

Wainscoat, R. J., Cohen, M., Volk, K., Walzer, H. J., \& Schwartz, D. E. 1992, ApJS, 83, 111 